\title{
Real-Time System Verification using P/T Nets *
}

\author{
Roberto Gorrieri ${ }^{\dagger} \quad$ Glauco Siliprandi ${ }^{\ddagger}$ \\ 'Dipartimento di Matematica, Università di Bologna \\ Piazza di Porta S. Donato 5, I-40127 Bologna, Italy \\ †Dipartimento di Matematica, Università di Siena \\ Via del Capitano 15, I-53100 Siena, Italy \\ e-mail:\{gorrieri,silipran\}@cs.unibo.it
}

\begin{abstract}
Timed Nets are proposed to model the behavior of real-time systems. Net transitions are annotated by timing constraints, using finitely many realvalued clocks. A timed net accepts timed words, i.e. infinite sequences in which a time of occurrence is associated with each symbol. We study expressiveness, closure properties and decision problems of such nets, where the acceptance condition is based on actions. The main result of the paper is an algorithm for deciding the inclusion problem for timed languages.
\end{abstract}

\section{Introduction}

There is a great variety of automata-based approaches to the specification and verification of real-time systems, depending mainly on the assumptions about the nature of time. The use of dense-time domains (events may happen arbitrarily close to each other) is an important feature whenever one is interested in modeling heterogeneous systems, i.e., systems composed of digital and analogical (hence, continuous) devices.

Among the models based on dense-time, particular interest has been stirred up by Timed Automata, proposed by Alur and Dill [1,2]. This model is essentially the timed version of finite-state $\omega$-automata, hence recognizing timed words - infinite sequences in which a real-valued time is associated with each symbol. A timed word is recognized by one of such automata if, for instance, the set of those states passed through infinitely often is equal to a given set of accepting states. The behavior of a real-time system is modeled by a timed language $\mathcal{L}_{\text {imp }}$; since also the requirements the system must satisfy can be expressed as a timed language $\mathcal{L}_{\text {spec }}$, the problem of verifying that a system satisfies a certain property essentially reduces to the inclusion problem of the implementation timed language $\mathcal{L}_{i m p}$ into the specification timed language $\mathcal{L}_{\text {spec }}$. Due to the decidability of the inclusion problem, Timed Automata have been profitably used for the automatic formal verification of real-time finite-state systems [2].

*This research has been partially supported by CNR grant N.92.00069.CT12.115.25585 and by MURST. 
Despite of their elegant characterization and strong properties, Timed $\mathrm{Au}$ tomata are not expressive enough to model systems with an infinite number of states. Here, we extend the approach of Alur and Dill to a more general class of automata, namely Place/Transition Petri Nets, where the number of tokens in each place can increase unboundedly. As the global state of a system is the collection of the tokens in the places, the number of global states of the system a $\mathrm{P} / \mathrm{T}$ net represents is infinite.

Our approach follows an action based acceptance condition: a timed word is accepted iff the set of those transitions fired infinitely often belongs to a given family of sets of transitions. The choice of such an acceptance condition is due to the fact that the set of transitions of a (Timed) $\mathrm{P} / \mathrm{T}$ Net is finite. This permits to prove some relevant decidability results: the problem of language emptiness and the problem of inclusion of a timed $\mathrm{P} / \mathrm{T}$ net language in a (deterministic) timed regular language. These are at the base of automatic verification of realtime properties, expressed as Timed Automata, of real-time systems, expressed as. Timed P/T Nets.

An example in Section 7 shows a simple real-time system which is modeled by a Timed P/T Net and which cannot be represented through a Timed Automaton, hence proving that the class of systems we can model is strictly larger. The final section introduces a simple example of application of our theoretical results.

\section{$2 \omega$-languages and $\omega$-automata.}

In this section we give some basic definitions about $\omega$-words and their recognizing automata. For more details see $[4,5]$.

Let $\Sigma$ be a finite alphabet. $\Sigma^{*}$ is the set of all finite sequences over $\Sigma$. An infinite sequence over $\Sigma$, also called $\omega$-word, is a map $\sigma: \mathbb{N}^{+} \rightarrow \Sigma$. Since $\sigma_{i}$ denotes the $i$ th symbol of $\sigma$, we also write $\sigma=\sigma_{1} \sigma_{2} \ldots$. The set of all $\omega$-words over $\Sigma$ is denoted by $\Sigma^{\omega}$. If $A$ is another alphabet, $h: \Sigma \rightarrow A$ and $\sigma \in \Sigma^{\omega}$, we use $h(\sigma)$ to denote the $\omega$-word $h\left(\sigma_{1}\right) h\left(\sigma_{2}\right) \ldots \in A^{\omega} . L \subseteq \Sigma^{\omega}$ will be called a $\omega$-language. With $\exists^{\infty} n . P(n)$ we denote a property $P$ which holds infinitely often: $\forall m \in$ $\mathbb{N} . \exists n>m . P(n)$ holds. We define $\operatorname{In}(\sigma) \stackrel{\text { def }}{=}\left\{a \in \Sigma \mid \exists_{i}^{\infty} . \sigma_{i}=a\right\}$.

In the literature various types of finite state $\omega$-automata have been studied: among these, we recall Büchi automata. A Transition Table (TT for short) is a tuple $\mathcal{A}=\left(\Sigma, S, S_{0}, E\right)$, where $\Sigma$ is an alphabet, $S$ is a finite set of states, $S_{0} \subseteq S$ is a set of start states, and $E \subseteq S \times S \times \Sigma$ is a set of transitions. A run $r=\left(s_{0}, s_{1}, \sigma_{1}\right)\left(s_{1}, s_{2}, \sigma_{2}\right) \ldots \in E^{\omega}$ on an $\omega$-word $\sigma$ is such that $s_{0} \in S_{0}$. The sequence of reached states in the run $r$ is the $\omega$-word $s_{1} s_{2} s_{3} \ldots \in S^{\omega}$, denoted by $S t(r)$. A Büchi Automata (BA for short) is a tuple $\mathcal{A}=\left(\Sigma, S, S_{0}, E, F\right)$ such that $\left(\Sigma, S, S_{0}, E\right)$ is a TT and $F \subseteq S$. A run $r$ is accepted iff $\operatorname{In}(S t(r)) \cap F \neq \emptyset$. The accepted $\omega$-language is $\mathcal{L}(\mathcal{A}) \stackrel{\text { def }}{=}\left\{\sigma \in \Sigma^{\omega} \mid\right.$ there is a run on $\sigma$ accepted by $\mathcal{A}\}$. The class of languages accepted by BAs, called regular $\omega$-languages, is denoted by $\mathcal{B A}$. 
Definition 2.1 (Place/Transition Petri Nets) $N=\left(\Sigma, P, T, F, W, h, m_{0}\right)$ is a PTP, where $\Sigma$ is an input alphabet, $P$ is a finite set of places, $T$ is a finite set of transitions (disjoint from $P), F \subseteq(P \times T) \cup(T \times P)$ is the flow relation, $W: F \rightarrow \mathbb{N}^{+}$is the weight function, $h: T \rightarrow \Sigma$ is the labeling function, $m_{0} \in \mathbb{N}^{P}$ is the initial marking (the functions from $P$ to $\mathbb{N}$, are called markings for $N$; such a set will be usually denoted by $M$ and ranged over by $m$ ).

The pre-set of a transition $t$ is ${ }^{\bullet} t \stackrel{\text { def }}{=}\{p \mid(p, t) \in F\}$, and its post-set is $t^{\bullet} \stackrel{\text { def }}{=}$ $\{p \mid(t, p) \in F\}$. $N$ has concession in $m$ iff $m(p) \geq W(p, t)$ for all $p \in \in^{\circ} t$. If $t$ has concession in $m$ then $t$ fires $m$ to $m^{\prime}$, where $m^{\prime}(p)=m(p)-W(p, t)$ if $p \in \in^{\bullet} t \backslash t^{\bullet}$, $m^{\prime}(p)=m(p)+W(t, p)$ if $p \in t^{\bullet} \backslash{ }^{\bullet} t, m^{\prime}(p)=m(p)-W(p, t)+W(t, p)$ if $p \in^{\bullet} t \cap t^{\bullet}$ and $m^{\prime}(p)=m(p)$ if $p \in P \backslash\left(\boldsymbol{\varphi} \cup t^{\bullet}\right)$. This relation on $M \times T \times M$ is the firing relation, denoted by $m(t) m^{\prime}$.

We say that $r \in T^{\omega}$ is a run of $N$ over $\sigma \in \Sigma^{\omega}$ iff $h(r)=\sigma$ and there are $m_{1}, m_{2}, \ldots \in M$ such that $\forall i \in \mathbb{N} . m_{i}\left(r_{i+1}\right) m_{i+1} . m_{0}, m_{1}, \ldots$ is the sequence of markings associated to $r$.

In [6] PTPs are provided with acceptance conditions based on transitions fired infinitely often in a run. Among the various notions proposed there, we consider the one for which the class of accepted languages is the largest.

Definition 2.2 (Action Based PTP) $N=\left(\Sigma, P, T, F, W, h, m_{0}, \mathcal{F}\right)$ is an ABP, where $\left(\Sigma, P, T, F, W, h, m_{0}\right)$ is a PTP and $\mathcal{F} \subseteq \mathfrak{p}^{+}(T)$. A run $r$ is accepted iff In $(r) \in \mathcal{F} . \mathcal{A B P}$ denotes the class of $\omega$-languages accepted by ABPs.

Theorem 2.3 [6] The emptiness problem is decidable for $\mathcal{A B P}$.

\section{Timed Languages}

In this section we present the timed languages, as introduced in [2].

Definition 3.1 (Time Sequence) A time sequence $\tau=\tau_{1} \tau_{2} \ldots$ is an infinite sequence of positive reals $\tau_{i} \in \mathbb{R}^{>0}$, satisfying

Monotonicity: $\tau_{i}<\tau_{i+1}$ for all $i \geq 1$.

Progress: for every $t \in \mathbb{R}^{\geq 0}$ there is some $i \geq 1$ such that $\tau_{i}>t$.

For any time sequence $\tau=\tau_{1} \tau_{2} \ldots$ we assume $\tau_{0} \stackrel{\text { def }}{=} 0$.

The progress condition is introduced to avoid the Zeno paradox: it will never be the case that infinitely many events happen in a bounded time interval.

Definition 3.2 (Timed Words and Languages) A timed word over $\Sigma$ is a pair $(\sigma, \tau)$ where $\sigma \in \Sigma^{\omega}$ and $\tau$ is a time sequence. $\Sigma^{t}$, ranged over by $\psi$, denotes the set of all timed words over $\Sigma$. A timed language is a set $L \subseteq \Sigma^{t}$.

The Untime operation discards the time values associated with the symbols, i.e., it considers the projection of a timed word $(\sigma, \tau)$ on the first component.

Definition 3.3 (Untimed Language) Given a timed language $L$, we define Untime $[L] \stackrel{\text { def }}{=}\{\sigma \mid \exists \tau .(\sigma, \tau) \in L\}$, also denoted by $\pi_{1}(L)$. 


\section{Timed Finite State Automata}

In $[1,2]$ the definition of finite state $\omega$-automata is augmented, so that they accept timed languages. In this section we recall some results presented there and a new result of ours.

Transition tables are extended to timed TTs so that they can read timed words. When executing a transition, the choice of the next state depends also on the time of the input symbol w.r.t. the times of the previously read symbols. For this purpose a finite set of clocks is associated with each TT. A clock can be set to zero simultaneously with the execution of a transition, while its value is equal to the time elapsed since the last time of reset. A clock constraint is associated with each transition and a transition may be taken only if the current values of the clocks satisfy its constraint.

Definition 4.1 (Clocks) For a set $C$ of clock variables, the set $\Phi(C)$ of clock constraints is defined inductively by

$$
\delta:=c \leq q|q \leq c| \neg \delta \mid \delta \wedge \delta
$$

where $c$ is a clock in $C$ and $q$ is a nonnegative rational constant. A clock interpretation (CI for short) for $C$ is a function $\nu: C \rightarrow \mathbb{R}^{\geq 0}$. We say that $\nu$ satisfies $\delta$ iff $\delta$ evaluates to true. $\nu_{0}$ denotes the CI such that $\forall c \in C . \nu_{0}(c) \stackrel{\text { def }}{=} 0$. If $t \in \mathbb{R}^{\geq 0}$, then $\nu+t$ is the CI mapping every clock $c$ to $\nu(c)+t$, and $t \cdot \nu$ the one assigning $t \cdot \nu(c)$ to each clock $c$. For $Y \subseteq C, \nu[t / Y]$ denotes the CI assigning $t$ to each $c \in Y$ and agreeing with $\nu$ over the other clocks.

A Timed Transition Table (TTT for short) is a tuple $\mathcal{A}=\left(\Sigma, S, S_{0}, C, E\right)$, where $C$ is a finite set of clocks, and $E \subseteq S \times S \times \Sigma \times 2^{C} \times \Phi(C)$ is the set of transitions. $\left(s, s^{\prime}, a, y, \delta\right)$ represents a transition from $s$ to $s^{\prime}$ on input symbol $a$. The set $y \subseteq C$ gives the clocks to be reset when executing this transition, and $\delta$ is a clock constraint to be satisfied. A run of $\mathcal{A}$ on $(\sigma, \tau)$ is an infinite sequence of transitions $r=\left(s_{0}, s_{1}, \sigma_{1}, y_{1}, \delta_{1}\right)\left(s_{1}, s_{2}, \sigma_{2}, y_{2}, \delta_{2}\right) \ldots$ such that $(i) s_{0} \in S_{0}$ and (ii) there are $\nu_{1}, \nu_{2}, \ldots$ CIs such that $\delta_{i+1}$ is satisfied by $\nu_{i}+\left(\tau_{i+1}-\tau_{i}\right)$ and $\nu_{i+1} \stackrel{\text { def }}{=}\left(\nu_{i}+\left(\tau_{i+1}-\tau_{i}\right)\right)\left[0 / y_{i+1}\right]$. We say that $\nu_{0}, \nu_{1}, \ldots$ is the sequence of CIs associated to $r$, and $S t(r) \stackrel{\text { def }}{=} s_{1} s_{2} s_{3} \ldots$ is the sequence of reached states.

\section{Definition 4.2 (Timed Büchi Automata)}

A TBA is a tuple $\mathcal{A}=\left(\Sigma, S, S_{0}, C, E, F\right)$, where $\left(\Sigma, S, S_{0}, C, E\right)$ is a TTT, and $F \subseteq S$. A run $r$ is accepted iff $\operatorname{In}(S t(r)) \cap F \neq \emptyset$. The accepted timed language is $\mathcal{L}(\mathcal{A}) \stackrel{\text { def }}{=}\left\{\psi \in \Sigma^{t} \mid \mathcal{A}\right.$ has an accepting run over $\left.\psi\right\}$. The class of timed languages accepted by TBAs, called timed regular languages, is denoted by $\mathcal{T} B \mathcal{A}$.

\section{Definition 4.3 (Timed Muller Automata)}

A TMA is a tuple $\mathcal{A}=\left(\Sigma, S, S_{0}, C, E, \mathcal{F}\right)$, where $\left(\Sigma, S, S_{0}, C, E\right)$ is a TTT, and $\mathcal{F} \subseteq p^{+}(S)$. A run $r$ is accepted iff $\operatorname{In}(S t(r)) \in \mathcal{F}$. The set of timed languages accepted by TMAs is denoted by $\mathcal{T} \mathcal{M A}$. 
Theorem 4.4 [2] (i) $\mathcal{T B A}=\mathcal{T} \mathcal{M A}$

(ii) $\mathcal{T B A}$ is closed under finite union and intersection.

In order to define a class of timed languages closed under all boolean operations, deterministic automata are investigated. A TMA $\left(\Sigma, S, S_{0}, C, E, \mathcal{F}\right)$ is called deterministic (DTMA) if and only if $\left|S_{0}\right|=1$ and for all $s \in S$, for all $a \in \Sigma$, for every pair of transitions of the form $\left(s,-, a,-, \delta_{1}\right)$ and $\left(s,-, a,-, \delta_{2}\right)$, we have that $\delta_{1} \wedge \delta_{2}$ is unsatisfiable. The class of timed languages accepted by DTMA is denoted by $\mathcal{D} \mathcal{T} \mathcal{M A}$.

Theorem 4.5 [2] (i) $\mathcal{D} T \mathcal{M A} \subset \mathcal{T} \mathcal{M A}$

(ii) $\mathcal{D} T \mathcal{M A}$ is closed under finite union, intersection and complementation.

We now study new acceptance conditions based on transitions rather than states. We define the action based timed Büchi automata and the action based timed Muller automata, and we prove that these have the same expressive power of TBAs and TMAs.

Definition 4.6 (Action Based TBA) $\mathcal{A}=\left(\Sigma, S, S_{0}, C, E, F\right)$ is an ABTBA, where $\left(\Sigma, S, S_{0}, C, E\right)$ is a TTT, and $F \subseteq E$ is the set of accepting transitions. A run $r$ is accepted iff $\operatorname{In}(r) \cap F \neq \emptyset$. The set of accepted timed languages is denoted by $\mathcal{A B T} \mathcal{B A}$.

Definition 4.7 (Action Based TMA) $\mathcal{A}=\left(\Sigma, S, S_{0}, C, E, \mathcal{F}\right)$ is an ABTMA, where $\left(\Sigma, S, S_{0}, C, E\right)$ is a TTT, and $\mathcal{F} \subseteq \wp^{+}(E)$ specifies an acceptance family. A run $r$ is accepted iff $\operatorname{In}(r) \in \mathcal{F}$. The set of timed languages accepted by ABTMAs is denoted by $\mathcal{A B T} \mathcal{M A}$.

Theorem $4.8[3] \mathcal{A B T} B \mathcal{A}=\mathcal{T} B \mathcal{A}=\mathcal{T} \mathcal{M A}=\mathcal{A B T} \mathcal{M A}$.

\section{Timed Place/Transition Nets}

In this section we augment the definition of PTPs following the same idea illustrated in the previous section, so that they can recognize timed words.

Definition 5.1 (Timed P/T Net) $N=\left(\Sigma, P, T, F, W, h, m_{0}, C, c^{c}, c^{r}\right)$ is a TP, where $N^{\prime}=\left(\Sigma, P, T, F, W, h, m_{0}\right)$ is a PTP, $C$ is a finite set of clocks, $c^{c}: T \rightarrow$ $\Phi(C)$ gives the clock constraint associated to each transition, and $c^{r}: T \rightarrow 2^{C}$ gives the set of clocks to be reset when a transition is executed.

A snapshot for $N$ is a couple $(m, \nu)$ such that $m$ is a marking for $N^{\prime}$ and $\nu$ is a CI for $C$. The set of snapshots is denoted by $\Gamma$. A transition $t$ of $N$ has concession in $(m, \nu)$ for $N$ iff $t$ has concession in $m$ for $N^{\prime}$ and $\nu$ satisfies $c^{c}(t)$. If $t$ has concession in $(m, \nu)$, then $t$ fires $(m, \nu)$ to $\left(m^{\prime}, \nu^{\prime}\right)$ where $m\left(t>m^{\prime}\right.$ and $\nu^{\prime}=\nu\left[0 / c^{r}(t)\right]$. This relation on $\Gamma \times T \times \Gamma$ is the timed firing relation denoted by $(m, \nu)(t\rangle\left(m^{\prime}, \nu^{\prime}\right)$. A run of $N$ over $(\sigma, \tau)$ is an infinite sequence $r=t_{1} t_{2} \ldots \in T^{w}$ such that $h(r)=\sigma$ and there are $\left(m_{1}, \nu_{1}\right),\left(m_{2}, \nu_{2}\right), \ldots$ snapshots for $N$ such that $\forall i \in \mathbb{N} .\left(m_{i}, \nu_{i}+\left(\tau_{i+1}-\tau_{i}\right)\right)\left(t_{i+1}\right)\left(m_{i+1}, \nu_{i+1}\right) .\left(m_{0}, \nu_{0}\right),\left(m_{1}, \nu_{1}\right), \ldots$ is the $s e-$ quence of snapshots associated to $r$. 


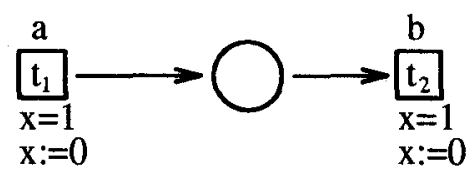

Figure 1: An example of timed $\mathrm{P} / \mathrm{T}$ net.

For example, consider the TP in Figure 1, where $a$ and $b$ are the labels for $t_{1}$ and $t_{2}$. In every run, in each moment of the execution, the number $m$ of fired $t_{1}$ transitions is not less than the number $n$ of executed $t_{2}$ transitions; indeed the place keeps exactly $m-n$ tokens. The timing constraints impose that a transition must be fired after each time unit, thus the first transition in the run fires at time 1 , the second at time 2 and so on.

We provide TPs with an action based acceptance condition.

Definition 5.2 (Action Based TP) $N=\left(\Sigma, P, T, F, W, h, m_{0}, C, c^{c}, c^{r}, \mathcal{F}\right)$ is an ATP, where $\left(\Sigma, P, T, F, W, h, m_{0}, C, c^{c}, c^{r}\right)$ is a TP and $\mathcal{F} \subseteq \wp^{+}(T)$ is an acceptance family. A run $r$ is accepted iff $\operatorname{In}(r) \in \mathcal{F}$. The accepted timed language is $\mathcal{L}(N) \stackrel{\text { def }}{=}\left\{\psi \in \Sigma^{t} \mid N\right.$ has an accepting run over $\left.\psi\right\}$. The class of accepted timed languages is denoted by $\mathcal{A} \mathcal{T} \mathcal{P}$.

Consider again the net in Figure 1, with acceptance family $\mathcal{F} \stackrel{\text { def }}{=}\left\{\left\{t_{1}\right\},\left\{t_{1}, t_{2}\right\}\right\}$ : it can be proved that every run is accepted and the recognized timed language is $\left\{(\sigma, \tau) \in\{a, b\}^{t} \mid \forall i \in \mathbb{N} . \#\left\{n \leq i \mid \sigma_{n}=a\right\} \geq \#\left\{n \leq i \mid \sigma_{n}=b\right\} \wedge \tau=123 \ldots\right\}$.

\section{Emptiness}

In this section we develop an algorithm that, given an ATP accepting a timed language $L$, constructs an $\mathrm{ABP}$ recognizing $U$ ntime $[L]$ (in [2] an analogous procedure was defined for TBAs). This important result allows us to state the decidability of the emptiness problem for timed languages in $\mathcal{A T} \mathcal{P}$, and defines a limit to the expressive power of ATPs, in the sense that a timed language may be accepted by an ATP only if its Untime is recognized by an ABP. We use this fact to prove the nonclosure of $\mathcal{A T P}$ under complementation.

First of all we want to prove that it suffices to consider those nets in which only integer constants are used in clock constraints.

\section{Definition 6.1 (Multiplied Clock Constraints)}

Given a TP $N$ and $t \in \mathbb{R}^{>0}, N_{t}$ is the TP obtained by replacing each constant $q$, in each $\delta$ appearing in $N$, by $t \cdot q$.

Lemma 6.2 Consider a TP $N$, a timed word $(\sigma, \tau)$, and a positive rational $t$. Then $r$ is a run of $N$ over $(\sigma, \tau)$ iff $r$ is a run of $N_{t}$ over $(\sigma, t \cdot \tau)$.

If we choose $t$ to be the least common multiple of all nonzero constants appearing in the clock constraints of $N$, then the clock constraints in $N_{t}$ use only nonnegative integers; furthermore, if $N$ is an ATP then $U$ ntime $[\mathcal{L}(N)]=U$ ntime $\left[\mathcal{L}\left(N_{t}\right)\right]$; 
as a consequence, $\mathcal{L}(N)=\emptyset$ iff $\mathcal{L}\left(N_{t}\right)=\emptyset$. Thus, in the remainder of the section we assume that clock constraints use nonnegative integers only.

The problem of checking the emptiness of the timed language recognized by an ATP $N$ can be more easily coped with if we show that this is equivalent to checking the emptiness of the language recognized by an ABP $N^{\prime}$. This could be done, in principle, by adding places for clock interpretations: a snapshot $\langle m, \nu\rangle$ in $N$ is simulated in $N^{\prime}$ by putting a token in the place corresponding to $\nu$. Even if the CIs for $N$ are infinite, we can define an equivalence relation $\cong$ on CIs, with a finite number of classes, and then add a new place for each of such classes. In $[2] \cong$ has been defined in the case of TBAs.

Definition 6.3 (Clock Regions) Let $q_{c}$ denote the largest integer $q$ such that $c \leq q$ or $q \leq c$ is a subformula of some $\delta$ appearing in the net $N$; for any nonnegative real $t, f r a c t(t)$ denotes the fractional part of $t$, and $\lfloor t\rfloor$ denotes the integer part of $t$; that is $t=\lfloor t\rfloor+f \operatorname{fract}(t)$.

$\nu \cong \nu^{\prime}$ iff the following three conditions hold:

1. $\forall c \in C \cdot\lfloor\nu(c)\rfloor=\left\lfloor\nu^{\prime}(c)\right\rfloor \vee\left(\nu(c)>q_{c} \wedge \nu^{\prime}(c)>q_{c}\right)$

2. $\forall c, d \in C \cdot\left(\nu(c) \leq q_{c} \wedge \nu(d) \leq q_{d}\right) \Rightarrow$

$$
\left(f \operatorname{ract}(\nu(c)) \leq f \operatorname{ract}(\nu(d)) \Leftrightarrow f \operatorname{ract}\left(\nu^{\prime}(c)\right) \leq f \operatorname{ract}\left(\nu^{\prime}(d)\right)\right)
$$

3. $\forall c \in C . \nu(c) \leq q_{c} \Rightarrow\left(\operatorname{fract}(\nu(c))=0 \Leftrightarrow \operatorname{fract}\left(\nu^{\prime}(c)\right)=0\right)$

It can be proved that $\cong$ is an equivalence relation. An equivalence class is called a clock region (CR for short). [ $\nu]$ denotes the $\mathrm{CR}$ which $\nu$ belongs to. The set of CRs is ranged over by $\alpha$.

Proposition 6.4 Let $c \in C, \nu \cong \nu^{\prime}$ and $t \in T$. Then

1. $\nu$ satisfies $c^{c}(t)$ iff $\nu^{\prime}$ satisfies $c^{c}(t)$

2. $\nu\left[0 / c^{r}(t)\right] \cong \nu^{\prime}\left[0 / c^{r}(t)\right]$

3. $\nu(c)=0$ iff $\nu^{\prime}(c)=0$ and $\nu(c)>q_{c}$ iff $\nu^{\prime}(c)>q_{c}$.

Thus in the remainder of the section we shall say that $[\nu]$ satisfies $c^{c}(t)$ if $\nu$ satisfies $c^{c}(t)$, and we shall use $[v]\left[0 / c^{r}(t)\right]$ instead of $\left[\nu\left[0 / c^{r}(t)\right]\right]$.

In [3] an algorithm producing a representative CI for each clock region is provided: $\bar{\alpha}$ denotes the representative of class $\alpha$. As a consequence

\section{Proposition 6.5}

1. The set of clock regions is finite.

2. If $\nu \cong \nu^{\prime}$ and $t>0$, then there exists $t^{\prime}>0$ such that $\nu+t \cong \nu^{\prime}+t^{\prime}$.

Then a new place is introduced for each clock region. When a transition fires, it puts a token in a clock region place. This means that the CI reached just after the firing of the transition belongs to the clock region corresponding to that place. Moreover, the previous proposition states that, if $\nu \cong \nu^{\prime}, \nu$ and $\nu^{\prime}$ visit the same clock regions as time progresses.

Definition 6.6 (Time-successor) A CR $\alpha^{\prime}$ is a time-successor of a CR $\alpha$ iff for each $\nu \in \alpha$ there exists $t>0$ such that $\nu+t \in \alpha^{\prime}$. 
In [3] we provide an algorithm building the representative of any time successor of a given $\mathrm{CR}$. Now we define the ABP recognizing $U n$ time $[\mathcal{L}(N)]$.

Definition 6.7 (Region Net) Given a TP $N=\left(\Sigma, P, T, F, W, h, m_{0}, C, c^{c}, c^{r}\right)$ we define the PTP $\mathcal{R}(N) \stackrel{\text { def }}{=}\left(\Sigma, P^{\prime}, T^{\prime}, F^{\prime}, W^{\prime}, h^{\prime}, m_{0}^{\prime}\right)$ where

- $P^{\prime} \stackrel{\text { def }}{=} P \cup\{\alpha \mid \alpha$ is a $C R$ for $N\}$

- $T^{\prime} \stackrel{\text { def }}{=}\left\{\left(t, \alpha, \alpha^{\prime}\right) \mid t \in T\right.$ and there is an $\alpha^{\prime \prime}$ time-successor of $\alpha$, such that $\alpha^{\prime \prime}$ satisfies $c^{c}(t)$ and $\left.\alpha^{\prime}=\alpha^{\prime \prime}\left[0 / c^{r}(t)\right]\right\}$

- $F^{\prime} \stackrel{\text { def }}{=}\left\{\left(p,\left(t, \alpha, \alpha^{\prime}\right)\right) \mid(p, t) \in F\right\} \cup\left\{\left(\left(t, \alpha, \alpha^{\prime}\right), p\right) \mid(t, p) \in F\right\} \cup$ $\left\{\left(\alpha,\left(t, \alpha, \alpha^{\prime}\right)\right) \mid\left(t, \alpha, \alpha^{\prime}\right) \in T^{\prime}\right\} \cup\left\{\left(\left(t, \alpha, \alpha^{\prime}\right), \alpha^{\prime}\right) \mid\left(t, \alpha, \alpha^{\prime}\right) \in T^{\prime}\right\}$

- $W^{\prime}\left(p,\left(t, \alpha, \alpha^{\prime}\right)\right) \stackrel{\text { def }}{=} \begin{cases}W(p, t) & \text { if }(p, t) \in F \\ 1 & \text { otherwise }\end{cases}$ $W^{\prime}\left(\left(t, \alpha, \alpha^{\prime}\right), p\right) \stackrel{\text { def }}{=} \begin{cases}W(t, p) & \text { if }(t, p) \in F \\ 1 & \text { otherwise }\end{cases}$

- $h^{\prime}\left(t, \alpha, \alpha^{\prime}\right) \stackrel{\text { def }}{=} h(t)$

- $m_{0}(p) \stackrel{\text { def }}{=} \begin{cases}m_{0}(p) & \text { if } p \in P \\ 1 & \text { if } p=\left[\nu_{0}\right] \\ 0 & \text { otherwise }\end{cases}$

In the net $\mathcal{R}(N)$, any reachable marking has one token in only one of the clock region places, while all the other $C R$ places are empty.

Definition 6.8 $(\mathcal{R}(N)$ Markings) Let $m$ be a marking of $N$. $\langle m, \alpha\rangle$ denotes the marking in $\mathcal{R}(N)$ such that $\langle m, \alpha\rangle(p) \stackrel{\text { def }}{=} m(p)$ if $p \in P,\langle m, \alpha\rangle(\alpha) \stackrel{\text { def }}{=} 1$, and $\langle m, \alpha\rangle(p) \stackrel{\text { def }}{=} 0$ otherwise.

Now we want to establish a correspondence between each run in $N$ and some run in $\mathcal{R}(N)$.

\section{Definition 6.9 (Run Projection)}

Let $r=t_{1} t_{2} \ldots$ be a run of $N$ on $(\sigma, \tau)$ and $\left(m_{0}, \nu_{0}\right),\left(m_{1}, \nu_{1}\right), \ldots$ be the sequence of snapshots associated to $r$. We define the run projection of $r$ to be $[r] \stackrel{\text { def }}{=}$ $\left(t_{1},\left[\nu_{0}\right],\left[\nu_{1}\right]\right)\left(t_{2},\left[\nu_{1}\right],\left[\nu_{2}\right]\right) \ldots$

Lemma $6.10[r]$ is a run of the region net $\mathcal{R}(N)$.

Because of the progress condition, every clock in a run is either reset infinitely often, or from a certain time onwards it increases unboundedly.

Definition 6.11 (Progressive Run) A run of $\mathcal{R}(N)$, with associated sequence of markings $\left\langle m_{0},\left[\nu_{0}\right]\right\rangle,\left\langle m_{1},\left[\nu_{1}\right]\right\rangle, \ldots$, is progressive iff $\forall c \in C, \exists^{\infty} i .\left(\nu_{i}(c)=\right.$ $\left.0 \vee \nu_{i}(c)>q_{c}\right)$.

Lemma 6.12 If $r$ is a run of $N$, then $[r]$ is a progressive run of $\mathcal{R}(N)$. 
Conversely, we now claim that, given a progressive run $r^{\prime}$ of $\mathcal{R}(N)$, a run $r$ of $N$ can be defined such that $r^{\prime}$ is its projection.

Lemma 6.13 If $r^{\prime}=\left(t_{1}, \alpha_{0}, \alpha_{1}\right)\left(t_{2}, \alpha_{1}, \alpha_{2}\right) \ldots$ is a progressive run of $\mathcal{R}(N)$ over $\sigma$, then there exist a time sequence $\tau$ and a run $r$ of $N$ over $(\sigma, \tau)$ such that $r^{\prime}=[r]$.

Finally we define the acceptance family. A set of accepting transitions has to ensure that a run in $\mathcal{R}(N)$ may be accepted only if it is progressive.

Definition 6.14 (Progressive Acceptor) A set $R \subseteq T^{\prime}$ is a progressive acceptor if and only if $\forall c \in C . \exists\left(t, \alpha^{\prime}, \alpha\right) \in R . \bar{\alpha}(c)=0 \vee \bar{\alpha}(c)>\dot{q}_{c}$.

Theorem 6.15 Given an $A T P N$, there exists an $A B P N^{\prime}$ such that $\mathcal{L}\left(N^{\prime}\right)=$ Untime $[\mathcal{L}(N)]$.

$N^{\prime}$ is $\mathcal{R}(N)$ provided with the acceptance family $\mathcal{F}^{\prime} \stackrel{\text { def }}{=}\left\{R^{\prime} \subseteq T^{\prime} \mid \pi_{\mathbf{1}}\left(R^{\prime}\right) \in \mathcal{F}\right.$ and $R^{\prime}$ is a progressive acceptor $\}$. As a consequence, the following holds

Theorem 6.16 The emptiness problem for ATPs is decidable.

Proof: $L=\emptyset$ iff Untime $[L]=\emptyset$; the thesis follows by Th.6.15 and 2.3.

\section{Expressiveness and Closure Properties}

In this section we compare ATPs with timed finite state automata and show some closure properties w.r.t. boolean operations.

Theorem 7.1 [3] $\mathcal{T} \mathcal{B A} \subset \mathcal{A} \mathcal{A P}$.

The basic idea underlying the proof is that every ABTMA can be simulated by an ATP: a place in the net corresponds to a state in the ABTMA. On the contrary, there exist nets recognizing non regular timed languages. A counterexample is illustrated by the net $N$ in Figure 1, with acceptance family $\mathcal{F} \stackrel{\text { def }}{=}\left\{\left\{t_{1}\right\},\left\{t_{1}, t_{2}\right\}\right\}$.

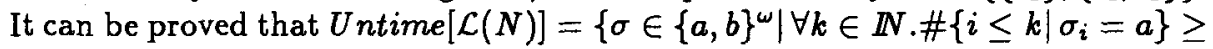
$\left.\#\left\{i \leq k \mid \sigma_{i}=b\right\}\right\}$ is not a regular $\omega$-language. In [2] Alur and Dill proved that this fact implies that $\mathcal{L}(N)$ is not a timed regular language.

Theorem $7.2[3] \mathcal{A T P}$ is closed under finite union and intersection.

$\mathcal{A T P}$ is not closed under complementation; consider the ATP $N$ in Figure 1 with no clock constraints, and acceptance family $\mathcal{F} \stackrel{\text { def }}{=}\left\{\left\{t_{1}\right\},\left\{t_{1}, t_{2}\right\}\right\}$. It can

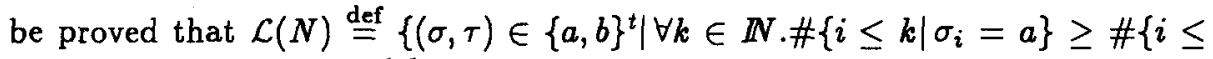

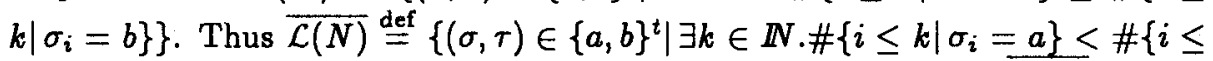

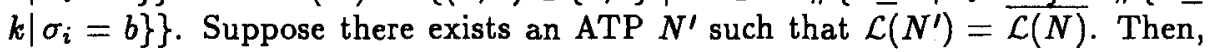
for Theorem 6.15 there exists an ABP $N^{\prime \prime}$ such that $\mathcal{L}\left(N^{\prime \prime}\right)=\left\{\sigma \in\{a, b\}^{\omega} \mid \exists k \in\right.$ $\mathbb{N}$.\#\{i $\left.\left.\leq k \mid \sigma_{i}=a\right\}<\#\left\{i \leq k \mid \sigma_{i}=b\right\}\right\}$, but it can be proved that such an ABP does not exist. 


\section{Verification}

Here we discuss how to use the theory of timed $\mathrm{P} / \mathrm{T}$ nets to prove correctness of some infinite-state real-time systems. A trace will be a timed word over sets of events: if two events $a$ and $b$ happen simultaneously, the trace will have the set $\{a, b\}$.

\section{Definition 8.1 (Timed Traces and Timed Processes)}

$\psi \in \mathfrak{p}^{+}(A)^{t}$ is a timed trace over alphabet $A$. A timed process is a pair $(A, L)$ where $A$ is a finite set of observable events, and $L$ is a timed language over $\mathfrak{p}^{+}(A)$. The set of timed processes is denoted by $\mathcal{T P}$. The class of processes modeled by ATPs is $\mathcal{A T P} \mathcal{P} \stackrel{\text { def }}{=}\{(A, L) \in \mathcal{T} \mathcal{P} \mid L \in \mathcal{A T P}\}$.

Remark 8.2 A property $\Pi$ can be represented as the set of traces satisfying it. Hence verifying that a process $P=(A, L)$ satisfies property $\Pi$ is equivalent to check that $L \subseteq \Pi$, i.e. that all the execution traces satisfy the property II.

Various operations can be defined on processes; these are useful for describing complex systems as composed of simpler ones. We will consider only parallel composition, which prescribes the joint behavior of a set of processes running concurrently.

The parallel composition operator can be conveniently defined using the projection operation. The projection of $(\sigma, \tau) \in \mathfrak{\wp}^{+}(A)^{t}$ onto $B \subseteq A$ is formed by intersecting each event set in $\sigma$ with $B$ and deleting all the empty sets from the sequence. Notice that the projection operation may result in a finite sequence, thus we define the set of timed traces projectable onto $B$.

Definition 8.3 (Projectable Timed Traces)

$A\left\lceil B \stackrel{\text { def }}{=}\left\{(\sigma, \tau) \in \mathfrak{\wp}^{+}(A)^{t} \mid \exists^{\infty} i . \sigma_{i} \cap B \neq \emptyset\right\}\right.$. If $B_{1}, \ldots, B_{n} \subseteq A$, we define $A \Gamma_{i} B_{i} \stackrel{\text { def }}{=}\left\{\psi \in \mathfrak{p}^{+}(A)^{t} \mid \wedge_{i} \psi \in A\left\lceil B_{i}\right\}\right.$.

Definition 8.4 (Projection) Assume $(\sigma, \tau) \in A\left\lceil B\right.$. We define $\xi_{1} \stackrel{\text { def }}{=} \min \{i \epsilon$ $\left.\mathbb{N}^{+} \mid \sigma_{i} \cap B \neq \emptyset\right\}, \xi_{k+1} \stackrel{\text { def }}{=} \min \left\{i>\xi_{k} \mid \sigma_{i} \cap B \neq \emptyset\right\}, \sigma^{\prime} \stackrel{\text { def }}{=} \sigma_{\xi_{1}} \sigma_{\xi_{2}} \ldots, \tau^{\prime} \stackrel{\text { def }}{=}$ $\tau_{\xi_{1}} \tau_{\xi_{2}} \ldots$, and $(\sigma, \tau)\left\lceil B \stackrel{\text { def }}{=}\left(\sigma^{\prime}, \tau^{\prime}\right)\right.$.

Definition 8.5 (Parallel Composition) Assume $P_{i}=\left(A_{i}, L_{i}\right)$ is a timed process for $i=1,2, \ldots, n$. Their parallel composition is the timed process $\|_{i} P_{i} \stackrel{\text { def }}{=}\left(\cup_{i} A_{i}, \|_{i} L_{i}\right)$ where $\|_{i} L_{i} \stackrel{\text { def }}{=}\left\{\psi \in\left(\cup_{i} A_{i}\right) \Gamma_{j} A_{j} \mid \wedge_{j} \psi\left\lceil A_{j} \in L_{j}\right\}\right.$.

We want to prove that $\mathcal{A T P \mathcal { P }}$ is closed under parallel composition. Assume $P_{i}=\left(A_{i}, L_{i}\right) \in \mathcal{A T P P}$ for $i=1,2, \ldots, n$, and $N_{i}$ is an ATP such that $\mathcal{L}\left(N_{i}\right)=$ $L_{i} . \mathcal{G}\left(L_{k}\right)$ denotes the timed language $\left\{\psi \in\left(\cup_{i} A_{i}\right)\left\lceil_{j} A_{j} \mid \psi\left\lceil A_{k} \in L_{k}\right\}\right.\right.$. The first step will be, for each $k \in\{1, \ldots, n\}$, to define an ATP $\mathcal{G}\left(N_{k}\right)$ such that $\mathcal{L}\left(\mathcal{G}\left(N_{k}\right)\right)=\mathcal{G}\left(L_{k}\right)$. 
Definition 8.6 (Generalization Construction)

Given the net $N_{k}=\left(\wp^{+}\left(A_{k}\right), P_{k}, T_{k}, F_{k}, W_{k}, h_{k}, m_{k}, c_{k}^{c}, c_{k}^{r}, \mathcal{F}_{k}\right)$, we define below the net $\mathcal{G}\left(N_{k}\right) \stackrel{\text { def }}{=}\left(\mathfrak{p}^{+}\left(\cup_{i} A_{i}\right), P_{k}, T_{k}^{\prime}, F_{k}^{\prime}, W_{k}^{\prime}, h_{k}^{\prime}, m_{k}, c_{k}^{\prime c}, c_{k}^{\prime r}, \mathcal{F}_{k}^{\prime}\right)$ as follows.

- $T_{k}^{\prime} \stackrel{\text { def }}{=} \bar{A}_{k} \cup\left\{(t, A) \mid A=\emptyset \vee A \in \bar{A}_{k}\right\}$ where $\bar{A}_{k} \stackrel{\text { def }}{=} \mathfrak{p}^{+}\left(\cup_{i} A_{i} \backslash A_{k}\right) ;{ }^{1}$

- $F_{k}^{\prime} \stackrel{\text { def }}{=}\left\{(p,(t, A)) \mid(p, t) \in F_{k}\right\} \cup\left\{((t, A), p) \mid(t, p) \in F_{k}\right\}$;

- $W_{k}^{\prime}(p,(t, A)) \stackrel{\text { def }}{=} W_{k}(p, t)$ and $W_{k}^{\prime}((t, A), p) \stackrel{\text { def }}{=} W_{k}(t, p)$;

- $h_{k}^{\prime}(A) \stackrel{\text { def }}{=} A$ and $h_{k}^{\prime}(t, A) \stackrel{\text { def }}{=} h_{k}(t) \cup A$.

- $c_{k}^{\prime c}(A) \stackrel{\text { def }}{=}$ true and $c_{k}^{\prime c}(t, A) \stackrel{\text { def }}{=} c_{k}^{c}(t)$;

- $c_{k}^{\prime r}(A) \stackrel{\text { def }}{=} \emptyset$ and $c_{k}^{\prime r}(t, A) \stackrel{\text { def }}{=} c_{k}^{r}(t)$;

- Given $T \subseteq T_{k}^{\prime}$, we say that $T$ is complete iff $\forall j . \exists t \in T . A_{j} \cap h_{k}^{\prime}(t) \neq \emptyset$. When $R \bar{\in} \mathcal{F}_{k}$, we say that $T$ emulates $R$ iff $\pi_{1}\left(T \backslash \bar{A}_{k}\right)=R$. Then $\mathcal{F}_{k}^{\prime} \stackrel{\text { def }}{=}\left\{T \subseteq T_{k}^{\prime} \mid T\right.$ is complete and there exists $R \in \mathcal{F}_{k}$ such that $T$ emulates $R$ \}.

It can be proved that $\mathcal{L}\left(\mathcal{G}\left(N_{k}\right)\right)=\mathcal{G}\left(L_{k}\right)$; since $\mathcal{A T} \mathcal{P}$ is closed under intersection, the following holds

Theorem 8.7 $\mathcal{A T P \mathcal { P }}$ is closed under parallel composition.

Typically, a system implementation is described as a composition of several components; if each component is a timed process modeled by an ATP and the specification is given as a property modeled by a DTMA, then the correctness of the implementation can be checked, due to the following

Theorem 8.8 Given $P_{i}=\left(A_{i}, \mathcal{L}\left(N_{i}\right)\right) \in \mathcal{A T P P}$, modeled by ATPs $N_{i}$ (for $i=1, \ldots, n)$, and a specification as a DTMA $\mathcal{A}$, the inclusion of $\|_{i} \mathcal{L}\left(N_{i}\right)$ in $\mathcal{L}(\mathcal{A})$ can be decided effectively.

For deciding if $\|_{i} \mathcal{L}\left(N_{i}\right) \subseteq \mathcal{L}(\mathcal{A})$, in fact, it suffices to check if $\|_{i} \mathcal{L}\left(N_{i}\right) \cap \overline{\mathcal{L}(\mathcal{A})}=$ $\emptyset$, and this can be done due to Theorems 8.7, 4.5, 7.2, and 6.16.

\section{A Verification Example}

As an example of automatic verification using ATPs, we consider a simple process to produce wooden black horses. In Figure 2 we show an ATP modeling a workman that carves a raw block of wood producing a wooden horse. We impose that both transitions must be fired infinitely often: thus the process ensures that if a new block is provided, then a new horse is refined within 2 minutes. Notice that the workman does not accept a new block until it has completely carved the previous one. This process could have been modeled by a TMA, too.

\footnotetext{
${ }^{1}$ If $A \in \bar{A}_{k}$, then events in $A$ can happen at any time, thus we introduce a new transition for each of such an $A_{;}$furthermore, events in $A$ can happen contemporaneously to events in $A_{k}$ hence, for each transition $t \in T_{k}$, we introduce a new transition $(t, A)$.
} 


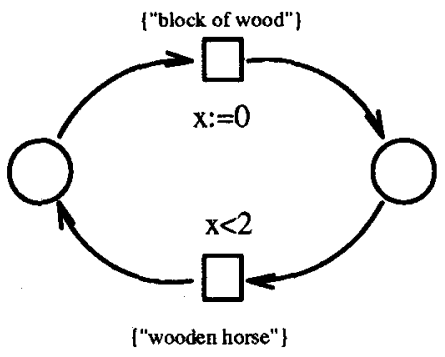

Figure 2: CARVER.

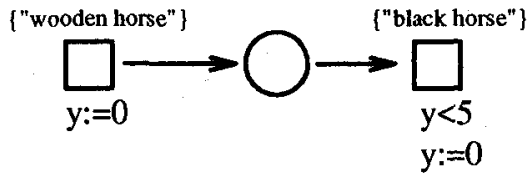

Figure 3: PAINTER.

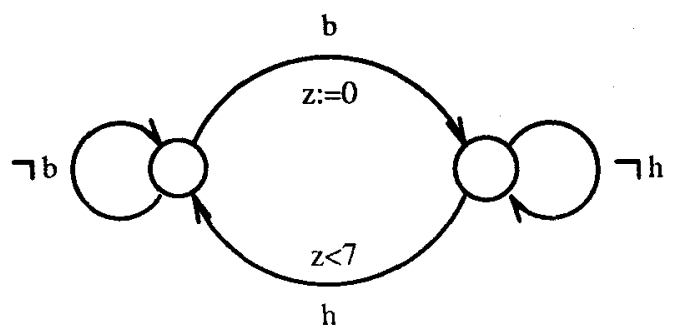

Figure 4: Strong Efficiency Property.

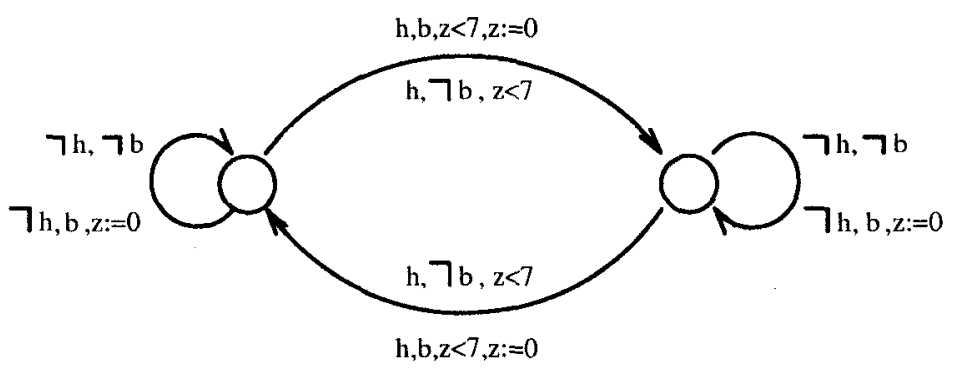

Figure 5: Weak Efficiency Property. 
In Figure 3 we show an ATP modeling a workman that obtains a wooden horse and paints it. Again, we impose that both transitions must be repeated infinitely often. The painter can accept more than one horse (we could say that he can put them on a shelf, and this operation makes him busy). However, if no new horse arrives for at least 5 minutes, then the workman has the time to concentrate on painting, and a black horse is produced. Since the process 'remembers' the number of carved horses that are still unpainted, this process cannot be modeled by a timed finite state automaton.

The complete process (Horse Manufactory) for the production of black horses is HM $\stackrel{\text { def }}{=}$ CARVER \| PAINTER. The factory administrator could be tempted to require that if a new block of wood is provided then, in no more than 7 minutes, a new black horse is produced. This property is modeled by the DTMA in Figure 4 (b denotes "block of wood", and h denotes "black horse"): a b-labeled edge stands for any event set containing $b$, and $a \neg b$-labeled edge means any event set not containing $b$. We impose that both states are reached infinitely often in an accepted run. Using Theorem 8.8, it can be proved that HM does not satisfy this property. On the other hand, the administrator could be pleased with the HM ensuring that if a new block is provided and for at least 7 minutes no new block is supplied, allowing all workmen to concentrate on the refining job, then at least a black horse is completed. This property is modeled by the DTMA in Figure 5 , in which a run is accepted only if it reaches both states infinitely often. Using Theorem 8.8 , it can be proved that HM satisfies this weaker property.

\section{References}

[1] R. Alur, D. Dill. "Automata for Modeling Real-Time Systems". In Proc. ICALP 90, LNCS 443, 322-335. Springer, 1990.

[2] R. Alur, D. Dill. "The Theory of Timed Automata". In Proc. of the REX workshop "Real-Time: Theory in Practice", LNCS 600, 45-73. Springer, 1992.

[3] R. Gorrieri, G. Siliprandi. "A Theory of P/T Nets for Timed Languages". In preparation.

[4] W. Thomas. "Automata on Infinite Objects". Handbook of Theoretical Computer Science. Elsevier, 133-191, 1990.

[5] R. Valk. "Infinite Behavior of Petri Nets". TCS 25, 311-341, 1983.

[6] R. Valk, M. Jantzen. "The Residue of Vector Sets with Applications to Decidability Problems in Petri Nets". Acta Informatica 21, 643-647, 1985. 